\title{
\#tolvaptan è ancora fermo ma noi NON stiamo fermi ad aspettare!
}

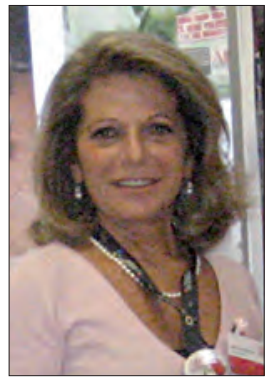

Luisa Sternfeld Pavia
II 27 maggio 2015, la Commissione europea autorizzava Jinarc (principio attivo tolvaptan) come primo farmaco in Europa "indicato per rallentare la progressione dello sviluppo di cisti e dell'insufficienza renale associata al Rene Policistico Autosomico Dominante (ADPKD) in adulti con CKD di stadio da 1 a 3 all'inizio del trattamento, con evidenza di malattia in rapida progressione".

A partire da quella data, ai pazienti di Inghilterra, Scozia, Germania, Francia, Belgio, Svizzera, Norvegia veniva dato accesso al farmaco. E in Italia?

In Italia i pazienti ancora aspettano tolvaptan, farmaco che AIFA ha scelto di non rimborsare nel marzo 2016, a distanza di un anno dalla richiesta di autorizzazione depositata dall'azienda Otsuka Pharmaceutical Italy.

AIRP, in data 16 marzo 2016, ha chiesto alle massime Istituzioni di dare accesso alle cure, inviando al Presidente della Repubblica, ad AIFA, alle autorità del Governo, Parlamento e ai Ministeri competenti la nostra petizione \#STOPalrenepolicistico che ha raccolto 5105 firme online.

Risultato? Nessuna risposta concreta a questa ingiustizia, che prevarica la decisione di una intera comunità medicoscientifica europea e nega il diritto alle cure nel nostro Paese.

\section{Ma noi NON siamo stati fermi ad aspettare!}

Il 28 aprile scorso AIRP ha incontrato la senatrice Biancone, autrice di una interrogazione parlamentare sulle intenzioni del Governo nei confronti dei pazienti con ADPKD, discussa successivamente, il 21 giugno, in Commissione Igiene e Sanità.

Molto si è detto a favore dei diritti dei pazienti con ADPKD ma nulla è cambiato nei fatti per l'accesso alle cure.

Accepted: November 24, 2016

Published online:December 14, 2016

Indirizzo per la corrispondenza:

Dr.ssa Luisa Sternfeld Pavia

AIRP Associazione Italiana Rene Policistico onlus

Via A. Bazzini 2

20131 Milano

luisa.sternfeld.airp@renepolicistico.it

\section{Ma noi NON siamo stati fermi ad aspettare!}

Lo scorso 10 ottobre, abbiamo indirizzato alcune domande all'azienda Otsuka che ci ha così risposto:

Quando è prevista la pubblicazione in GU (Gazzetta Ufficiale) del farmaco in classe C RNRL e quali sono le azioni future?

"AIFA ha deciso di classificare la specialità medicinale Jinarc in classe C (non rimborsata) RNRL. Tale classificazione, di fatto, andrebbe a limitare l'accesso alla terapia solo ed esclusivamente a quei pazienti che possono permettersi di acquistare il farmaco, creando, quindi, un'evidente disuguaglianza non solo tra pazienti italiani e pazienti di altre nazioni europee, ma anche tra i pazienti italiani di diverso ceto sociale. Pertanto, con l'intenzione di rendere disponibile e accessibile il farmaco nel più breve tempo possibile, e dopo un'attenta analisi del processo di valutazione condotto da AIFA, l'azienda ha presentato ricorso al Tar del Lazio. L'esito di tale ricorso è atteso dopo il 23 novembre 2016, data dell'udienza in cui il Tar del Lazio si esprimerà nel merito. Le azioni future dipenderanno dall'esito del ricorso".

Quali rassicurazioni possono avere i pazienti, che hanno iniziato un trial, di poter proseguire con il farmaco a titolo gratuito come era stato promesso?

"Per i pazienti attualmente in sperimentazione nei diversi studi in corso in Italia, l'azienda garantirà la continuità del trattamento. Infatti, i pazienti provenienti dai diversi studi conclusi e che, a giudizio del clinico, possono trarre beneficio dal prolungamento del trattamento, saranno accolti in uno studio di estensione che, come da protocollo, garantirà il farmaco fino al termine del periodo di trattamento stesso".

Per finire, nel corso del Congresso Nazionale della SIN (Società Italiana di Nefrologia) che si è svolto a Milano nei giorni 12-15 ottobre, abbiamo chiesto ai Nefrologi italiani di firmare anche loro la petizione \#STOPalrenepolicistico. Ebbene, centodieci Nefrologi tra i più importanti esperti in Italia hanno firmato la petizione, esprimendo solidarietà nei confronti dei pazienti e disappunto per la decisione delle autorità di negare loro l'accesso all'unica terapia approvata di dimostrata efficacia.

AIFA è ferma, tolvaptan è fermo, ma la malattia non si ferma! ...e noi?

Noi NON staremo fermi ad aspettare! Noi NON possiamo più stare fermi ad aspettare... perché nel frattempo i nostri pazienti vanno in dialisi... 